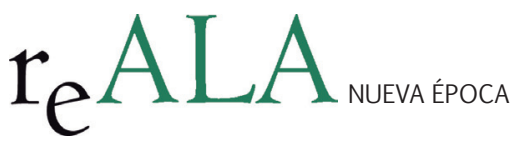

REALA, n 4, julio-diciembre 2015 ISSN: 1989-8975

DOI: http://dx.doi.org/10.24965/reala.voi4.10306

\title{
Incorporación de valor social a la contratación pública en España: Situación y perspectivas
}

\author{
Marta Solorzano García \\ UNED \\ msolorzano@cee.uned.es \\ Julio Navío Marco \\ UNED \\ jnavio@cee.uned.es \\ Raúl Contreras Comeche \\ Universidad de Valencia \\ rcontreras@nitua.eu
}

\section{Resumen}

La consideración del valor social en las transacciones con capital público debería ser un garante de mejora en las sociedades actuales. La inclusión de un reconocimiento e impulso de iniciativas de tipo social o medioambiental, por parte de las entidades públicas, en el momento de llevar a cabo concursos, compras o concesión de subvenciones, podría generar un incremento de la calidad de vida de los ciudadanos, objetivo que, sin duda, debería perseguir cualquier Administración Pública. En el presente artículo se revisa de forma comprensiva la situación de las administraciones (local, autonómica y nacional) en España, a la luz de los recientes cambios en la legislación comunitaria sobre contratación pública $y$, tras elaborar un diagnóstico de situación, se establecen recomendaciones para impulsar la incorporación de valor social a la contratación pública en España.

Palabras clave

Valor social, contratación pública, impacto social.

\section{Social value incorporation to public procurement in Spain: Situation and prospects}

\begin{abstract}
The consideration of social value in transactions with public capital should be a guarantee of improvement in our society. The recognition, inclusion and promotion of initiatives of social or environmental character, by public entities, at the time of conducting contests, procurement or grants, could generate an increase in the quality of life of citizens, target that certainly any public administration should pursue. In this article we review comprehensively the situation of the public administrations (local, regional and national) in Spain, and the recent changes in EU public procurement legislation, and after a diagnosis of situation, we develop several recommendations to encourage the incorporation of social value into the public procurement in Spain
\end{abstract}

Keywords

Social value, public procurement, social impact. 
La creación de valor por una organización se produce en tres áreas simultáneamente: área económica, área social y área socioeconómica. El valor económico se origina cuando se genera retorno financiero en una inversión. El valor social se crea cuando los recursos, procesos y políticas se combinan para generar mejoras en la vida de las personas o de la sociedad en conjunto. Y las organizaciones crean valor socioeconómico cuando sus actuaciones suponen ahorros de costes o ingresos para el sector público o la comunidad. En general, estos ahorros de costes o ingresos se producen como consecuencia de reducciones del gasto público social o incrementos en los impuestos cobrados.

La incorporación del concepto de valor social en la legislación pretende reconocer e impulsar iniciativas sociales que favorezcan el bienestar de aquellos colectivos o personas menos favorecidas, así como la promoción de su reinserción o inclusión en la sociedad y la promoción de valores solidarios y de carácter medioambiental.

La crisis económica ha provocado la necesidad de revisar los procesos de gestión públicos y privados en términos de eficiencia y del valor económico, social y medioambiental que aportan a los distintos grupos de interés. Además, nos encontramos en un momento en el que se buscan formas innovadoras de mejorar la compra y contratación pública (Edler y Georghiou, 2007; Thai, 2001), siendo cada vez más relevante la responsabilidad social y la sostenibilidad en los distintos niveles de la Administración Pública.

La inclusión del valor social es un hecho relevante, dada su función de instrumento privilegiado de intervención en la vida económica, política y social de los países, que supera con mucho la forma tradicional de ser un mero medio para comprar bienes o servicios, y satisfacer, de esta forma, los intereses generales de los ciudadanos (Araujo et al. 2005; Medina Jurado, 2010). Según Domínguez (2013), el problema radica en encontrar el equilibrio entre esa utilización de las compras públicas y el necesario respeto en los procedimientos públicos de contratación de los principios de igualdad de acceso a las licitaciones, libre competencia, objetividad, transparencia y publicidad. Habría que evitar el riesgo de utilización de prácticas proteccionistas amparadas por la contratación pública.

En un estudio preliminar de las diferentes variantes de la contratación social, Mc Crudden (2004) plantea varias preguntas, dos de los cuales son de particular relevancia: en primer lugar, la relación entre adquisición "verde" y contratación social, planteando el grado en que estos dos ámbitos se solapan o complementan. ¿El término general "contratación sostenible" nos ayuda a comprender los aspectos comunes de la contratación pública social y verde, o servir sólo para camuflar sus diferencias esenciales? En segundo lugar, la medida en la que la contratación social plantea diferentes cuestiones jurídicas y políticas respecto a su compatibilidad con los marcos jurídicos de las múltiples organizaciones internacionales y regionales.

Más allá de los problemas asociados con el volumen de la contratación pública, la literatura existente ha centrado su atención en una serie de cuestiones importantes, como la tendencia de la contratación pública a favorecer a empresas locales en la adjudicación, la creciente importancia de la colaboración entre el sector privado y público (Erridge y Greer, 2002)., y los procesos de licitación y contratación del gobierno para productos o servicios concretos (Brammer y Walker, 2011).

En el mismo sentido, en España hasta el momento se han abordado como objeto de investigación académica aspectos parciales del tema como la compra "sostenible" o contratación ecológica "verde" (Alfonso, 2008), revisado algunos casos pioneros en Gobiernos autonómicos y Administraciones locales (Bernete, 2013), o realizado estudios pormenorizados de Administraciones Públicas concretas (Aréjula y Azurmendi, 2014)

En el resto del artículo abordaremos de forma comprensiva y sistémica la situación existente en el ámbito europeo y nacional, realizaremos un diagnóstico de dicha situación y propondremos diversas recomendaciones para mejor incorporar el valor social a la contratación pública en España.

\section{INCORPORACIÓN DEL VALOR SOCIAL EN LA CONTRATACIÓN PÚBLICA: SITUACIÓN EN EUROPA Y ESPAÑA}

En el ámbito europeo se percibe un clima de cambio en la compra pública (Bovis, 2012) que posibilita la incorporación del valor social. El marco legislativo europeo recoge dicha incorporación en dos de sus directivas: la Directiva 2004/18/CE, de 31 de marzo de 2004, "sobre coordinación de los procedimientos de adjudicación de los contratos públicos de obras, de suministro y de servicios" y en la Directiva del Parlamento Europeo y del Consejo 2014/24/EU, de 26 de febrero de 2014, relativa a la Contratación Pública.

La primera de estas dos directivas está basada en la jurisprudencia del Tribunal de Justicia Europeo relativa a los criterios de adjudicación, y que "clarifica las posibilidades con que cuentan los poderes adjudicadores para atender las necesidades de los ciudadanos afectados, sin excluir el ámbito medioambiental o social”. 
Sin embargo, esta directiva limita la consideración de estos criterios sociales/medioambientales a su estricta vinculación al objeto del contrato, no otorgando al poder adjudicador una libertad de elección ilimitada. Por su parte, la segunda de las directivas ${ }^{1}$ a las que hacemos referencia, determina el obligatorio respeto, en la adjudicación de contratos públicos por las autoridades, de los principios del Tratado de Funcionamiento de la Unión Europea. En ella se considera que los Estados miembros han de poder reservar el derecho a participar en los procedimientos de adjudicación de contratos públicos (o lotes de los mismos) a talleres o empresas que desempeñen un papel social o "cuyo objetivo principal es apoyar la integración social y profesional o la reintegración de personas discapacitadas o desfavorecidas, como los desempleados, los miembros de comunidades desfavorecidas u otros grupos que de algún modo están socialmente marginados".

Junto a esta directiva, podemos, considerar como conjunto legislativo más favorable para la implantación de estas directrices de inclusión de aspectos de política social, ética y ambiental en los procedimientos de contratación pública, el creado tanto por la Estrategia Europa 2000 como por la Directiva 2014/24/EU.

La base de la Estrategia Europa son tres prioridades interrelacionadas y que se refuerzan mutuamente:

- El desarrollo de una economía basada en el conocimiento y la innovación.

- La promoción de una economía con pocas emisiones de carbono, eficaz en el uso de los recursos y competitiva.

- El fomento de una economía con alto nivel de empleo que tenga cohesión social y territorial.

La aprobación de la mencionada Directiva vino motivada por la necesidad de modernizar las normas vigentes sobre la contratación pública para, entre otros objetivos, garantizar la seguridad jurídica, "incrementar la eficiencia en el gasto público, facilitar la participación de las pequeñas y medianas empresas y permitir que los poderes adjudicadores empleen mejor la contratación púbica en apoyo a objetivos sociales comunes", fijándose su segundo objetivo como la permisión de que "los compradores utilicen mejor la contratación pública en apoyo de objetivos sociales comunes como la protección del medioambiente, una mayor eficiencia energética y en el uso de los recursos, la lucha contra el cambio climático, la promoción de la innovación, el empleo y la integración social y la prestación de servicios sociales de alta calidad en las mejores condiciones posibles".

La Directiva 2014/24/EU establece, asimismo, que "Ios Estados miembros deben disponer de amplias facultades discrecionales para organizar la elección de los proveedores de servicios"... "imponiendo únicamente el respeto de los principios fundamentales de transparencia e igualdad de trato". La razón para ello es el reconocimiento de que "estos servicios se prestan normalmente en un contexto específico que varía mucho de un Estado miembro a otro, debido a la existencia de distintas circunstancias administrativas, organizativas y culturales" y de que "los servicios tienen, por naturaleza, una dimensión transfronteriza muy limitada".

La contratación pública es un mecanismo fundamental para que las autoridades públicas apoyen la misión de las empresas sociales. De hecho, los análisis empíricos reflejan la importancia de las ventas de las empresas sociales a los organismos públicos oficiales. (Laville et al. 2006).

A este respecto, los diferentes países europeos presentan realidades diversas (Schulten et al. 2012). De acuerdo con Defourny y Nyssens, quienes definen las empresas sociales como "organizaciones privadas sin finalidad lucrativa que generan bienes y servicios relacionados directamente con su objetivo explícito de beneficiar a la comunidad. Se basan en dinámicas colectivas que involucran a diversos tipos de grupos de interés en sus órganos de gobierno, valoran su autonomía y soportan riesgos económicos vinculados por su actividad", (Defourny y Nyssens, 2008, p. 204) en muchos países europeos, el marco legal que regula la contratación y compra pública es poco favorable a las empresas sociales. Y entre estos países se encuentran Suecia, Irlanda, Reino Unido, Portugal y España. Pero, por otra parte, la legislación está evolucionando en otras naciones (como Bélgica), que empiezan a considerar activamente la introducción de cláusulas sociales en los concursos públicos. De hecho, las buenas prácticas nacionales y regionales en esta materia son relativamente variadas en toda la Unión Europea. Competir en el mercado con empresas con ánimo de lucro, utilizando exclusivamente criterios financieros parece a menudo difícil para las empresas sociales, que frecuentemente soportan costes que se externalizan en las empresas tradicionales, y algunas de estas empresas sociales, por lo tanto, postulan que las autoridades públicas deberían tener en cuenta su dimensión social en la adjudicación de contratos públicos.

En lo que respecta a la legislación española, las dos disposiciones relacionadas con la contratación pública en las que se contempla la inclusión o consideración de la valoración social en el momento de la adjudicación son:

1 Se puede consultar en http://ec.europa.eu/internal_market/publicprocurement/modernising_rules/reform_proposals/index_ en.htm

REALA, n 4, julio-diciembre 2015, ISSN: 1989-8975 - DOI: http://dx.doi.org/10.24965/reala.voi4.10306 
- La Ley 30/2007, de 30 de octubre, de Contratos del Sector Público y

- El Real Decreto Legislativo 3/2011, de 14 de noviembre, por el que se aprueba el texto refundido de la Ley de Contratos del Sector Público².

En la exposición de motivos de la Ley 20/2007, que incorpora las directrices de la Directiva europea 2004/18/ CE, se amplía el marco legislativo favorable y se hace mención a la inclusión de "sustanciales innovaciones en lo que se refiere a la preparación y adjudicación de los negocios sujetos a la misma". Dichas novedades hacen referencia a "la previsión de mecanismos que permiten introducir en la contratación pública consideraciones de tipo social y medioambiental, configurándolas como condiciones especiales de ejecución del contrato o como criterios para valorar las ofertas, prefigurando una estructura que permita acoger pautas de adecuación de los contratos a nuevos requerimientos éticos y sociales". Y en relación con los criterios medioambientales: "y que permitan ajustar la demanda pública de bienes y servicios a la disponibilidad real de los recursos naturales".

Por su parte, el Real Decreto 3/2011, en su artículo 150, recoge que, en lo referente a los criterios de valoración de las ofertas, se seguirán criterios directamente vinculados al objeto del contrato, incluyendo dentro de ellos la calidad, el precio o el valor técnico, pero también las características medioambientales o aquellas que puedan estar relacionadas con "la satisfacción de exigencias sociales que respondan a necesidades, definidas en las especificaciones del contrato, propias de las categorías de población especialmente desfavorecidas a las que pertenezcan los usuarios o beneficiarios de las prestaciones a contratar".

Lo que deja de manifiesto la intención de reconocer el valor social en la adjudicación de contratos por parte de la Administración Pública española.

La consideración de incorporar medidas de responsabilidad social en la contratación administrativa en el ámbito autonómico o provincial es, en España, todavía escasa. Algunos ejemplos de este esfuerzo son los llevados a cabo por la Diputación de Granada, la Diputación foral de Navarra, la Diputación Foral de Guipúzcoa, el Gobierno Vasco, la Generalidad de Catalunya, la Junta de Andalucía, la Junta de Extremadura y la Junta de Castilla y León.

Con la citada intención, la Diputación de Granada publicó en 2010, a través de su Delegación de Promoción Económica y Empleo, la "Guía Práctica para la incorporación de Cláusulas Sociales en la Contratación Pública". Asimismo, el desarrollo del subprograma "Responsabilidad Social y Compra Pública Responsable" y del proyecto "Granada empleo", tiene como principal objetivo para esta Administración facilitar el acceso al empleo a las personas y colectivos en situación desfavorecida.

La legislación de la Comunidad Foral de Navarra, concretamente la Ley Foral 6/2006, de 9 de junio, de contratos públicos, en su artículo 21, estableció que en "toda contratación se buscará la máxima eficiencia en la utilización de los fondos públicos y en el procedimiento, atendiendo a la consecución de objetivos sociales y de protección ambiental cuando guarden relación con la prestación solicitada y comporten directa o indirectamente ventajas para la entidad contratante" y, en relación con lo expuesto en este artículo, se fijó que las entidades contratantes podrían "incluir requerimientos pormenorizados de carácter social o medioambiental en la ejecución de los contratos". De esta forma, se entiende la intención de contemplar cláusulas sociales, siempre bajo la condición, ya recogida por la legislación nacional, de su necesaria relación con el objeto del proyecto.

Por su parte, la Diputación Foral de Guipúzcoa, de una forma mucho más explícita que en los dos últimos casos, y para promover la compra e inversión pública socialmente responsable, ha incorporado cláusulas sociales en todas sus contrataciones y las de todas sus sociedades dependientes. La regulación de este requerimiento se rige, desde el año 2013, a través de la Norma Foral 4/2013, de 17 de julio, de incorporación de cláusulas sociales en los contratos de obras del Sector Público Foral. A través de dicha norma se promueve la ayuda a personas en riesgo o situación de exclusión social, la eliminación de la discriminación laboral por sexo y la promoción de un empleo de calidad o se tienen en consideración aspectos medioambientales como la eficiencia energética, el fomento de la utilización de productos locales o incluso, la disminución en la producción de residuos. También se contemplan penalizaciones de tipo económico para el caso de incumplimiento de estos criterios en la contratación, lo que se entiende como un caso de claro de involucramiento, por parte de esta Administración provincial.

En un peldaño superior al de la Diputación de Guipúzcoa, el Gobierno Vasco, había aprobado cinco años antes la Resolución 6/2008, de 2 de junio, del director de la Secretaría del Gobierno y de Relaciones con el Parlamento, por la que se disponía la publicación del Acuerdo adoptado por el Consejo de Gobierno «sobre incorporación de criterios sociales, ambientales y otras políticas públicas en la contratación de la Administración de la Comunidad Autónoma y de su sector público».

2 Previos al Real Decreto 2011 y al nuevo espíritu de la Directiva 2014, existen numerosos estudios y destacables análisis a nivel nacional en la materia. Entre otros: Zabalegui (2002); Alonso-Alegre, G. (2009); Brunete, M.T. (2010); Zabalegui (2010). 
La Generalitat de Catalunya editó (en 2010 y versión revisada en 2012), una guía para la inclusión de cláusulas contractuales de carácter social, como respuesta a la preocupación por los aspectos sociales, puesta de manifiesto, inicialmente, a nivel europeo. En ella se exponen modelos y ejemplos de cláusulas de tipo social de recomendable introducción en el ámbito de la actividad contractual ordinaria.

En el mismo sentido, la reglamentación catalana, concretamente a través de la Ley 3/2015, de 11 de marzo, de medidas fiscales, financieras y administrativas (Diari Oficial de la Generalitat de Catalunya, 13/3/2015), en su disposición adicional decimocuarta, establece, en relación con los contratos por servicios sociales de atención a las personas, que "el Gobierno debe impulsar, de acuerdo con la normativa en materia de contratación pública, la inclusión de cláusulas sociales en todos los contratos de la Administración de la Generalidad y de su sector público que tengan por objeto servicios sociales de atención a las personas".

Es, en el ámbito autonómico de Andalucía, el Artículo 116 de su Ley 18/2003, de 29 de diciembre, por la que se aprueban medidas fiscales y administrativas, el que hace referencia a la reserva de contratos a centros, entidades de carácter social y empresas. En él se fija el porcentaje de adjudicación a "centros especiales de empleo y a entidades sin ánimo de lucro inscritas en los correspondientes registros oficiales de la Comunidad Autónoma de Andalucía, siempre que la actividad de dichos centros y entidades tenga relación directa con el objeto del contrato", así como las condiciones a cumplir, en función del procedimiento, el importe total del contrato y las características de las empresas adjudicatarias (número de trabajadores, grado de minusvalía de los mismos y tipología de sus contratos, entre otras).

La Junta de Extremadura, por su parte, a través de la Consejería de Empleo, Mujer y Políticas Sociales, ratificó a 1 de agosto de 2014, el Acuerdo de Consejo de Gobierno de 8 de julio de 2014, por el que se aprobaban las "directrices de política general dirigidas a los órganos de contratación de la Administración de la Comunidad Autónoma de Extremadura sobre contratos reservados e incorporación de cláusulas sociales en la contratación pública". De esta forma, la Junta extremeña entendía que su Administración debe satisfacer las necesidades de los ciudadanos para los que trabaja, y sus mecanismos de contratación han de contribuir "a la consecución de otros objetivos sociales, económicos, éticos o ambientales, como una herramienta jurídica al servicio de los poderes públicos para el cumplimiento de sus fines o sus políticas públicas y ello, en la medida en que sea acorde tanto con la legislación vigente como con la necesaria eficacia y austeridad que ha de predicarse del uso de fondos públicos, en este momento, especialmente limitados".

En el caso de la Comunidad Autónoma de Castilla y León, el ejemplo más patente de la inclusión del "valor social" en la contratación es el Acuerdo 59/2012, de 26 de julio, de la Junta de Castilla y León (BOCYL n. 147/2012), según el cual su Consejería de Hacienda, aprobaba directrices vinculantes para aquellos órganos de contratación de la Administración de dicha comunidad "relacionados con la incorporación de cláusulas sociales en la contratación pública". Entendiendo por cláusulas sociales aquellas "que establecen la inclusión de aspectos de política social en los procedimientos de contratación pública como el fomento del empleo de personas en situación o riesgo de exclusión social, la inserción laboral de personas con discapacidad, el fomento de la estabilidad en el empleo, la promoción de la igualdad efectiva entre mujeres y hombres y el impulso de medidas de conciliación de la vida familiar y laboral en las empresas".

A nivel local, localidades como: Salamanca, Lleida, Burlada, Madrid, Cornellá, Pamplona, Alpedrete, Alcañiz, Alcazar San Juan, A Coruña, Aretxabaleta, Avilés, Burgos, Castellón, Ceuta, Jaén, Getxo, Las Gabias, Manresa, Puerto Real, Mallorca, Amurrio, Getafe, Vitoria Gasteiz, Alcazar San Juan, Girona, Ondarroa, Paterna o SantBoi, han llevado a cabo esfuerzos para la regulación e incorporación de criterios sociales en sus contrataciones. Su análisis detallado se estima en este punto innecesario, debido a la gran diversidad de redacciones normativas y multiplicidad de casos.

Actualmente hay una gran cantidad de piezas legislativas, especialmente a nivel local, para la aplicación de cláusulas de tipo social a los contratos. Las causas probables de esta gran diversidad dentro de cada una de las diferentes fórmulas legislativas pueden venir derivadas de la falta de existencia de medidas concretas fijadas por la legislación nacional y autonómica. Como hemos visto con anterioridad, su consideración es patente pero en ningún caso se fijan baremos, porcentajes, cláusulas idénticas o se establecen unos criterios precisos para su aplicación en cada uno de los supuestos generales de un tipo de adjudicación concreta. La gran disparidad de contratos a los que se puede aplicar el clausulado social, la diferencia de problemáticas ciudadanas en la realidad de cada localidad y la disparidad en la cuantía económica de los contratos, en función de cada Administración local, pueden ser ejemplo de las causas de la gran heterogeneidad respecto a las bases reglamentarias de cada localidad en la determinación de los criterios de "reserva social" que se dan en cada uno de sus contratos. 
Unida a esta ingente cantidad de piezas legislativas, se manifiesta la diversidad existente en las posibilidades de incluir las cláusulas sociales en los procesos de contratación pública. Ésta se puede llevar a cabo, por ejemplo, a través de la reserva de contratos. Estos contratos reservados se califican como aquellos susceptibles de proporcionar un beneficio social, tal vez proporcionando empleo, a un grupo de personas a los que les suponga difícil la obtención del mismo, por su situación marginal o de exclusión social. También se pueden incluir las cláusulas fijando los criterios de adjudicación, estipulando criterios de ejecución o a través de la redacción de instrucciones, circulares, modelos integrales. Asimismo, la reserva de contratos inferiores a un límite económico fijado es una fórmula aplicable para la consideración de estas cláusulas sociales, ya que puede favorecer la participación de entidades de menor tamaño.

\section{DIAGNÓSTICO Y RECOMENDACIONES}

Fruto del análisis efectuado, hemos podido a identificar dos características respecto a la forma en la que la Administración Pública española incorpora el valor social en la contratación pública: la gran cantidad de piezas legislativas y la heterogeneidad. Las cuales generan dos claras consecuencias:

- Una importante carga de trabajo para las Administraciones Locales.

- Desigualdades para los ciudadanos, dependiendo del municipio o Comunidad Autónoma de la que sean habitantes.

El entorno socio-económico actual es el que ha favorecido el desarrollo de la consideración del "valor social" en los procedimientos de adjudicación pública y su incorporación a partir de las pautas marcadas por la legislación europea. El agravamiento general, especialmente en la realidad de nuestro país, de la situación de empleabilidad de los colectivos más desfavorecidos y el incremento del número de personas que forman estos colectivos, unidos a una reducción en los presupuestos de las Administraciones Públicas y una concentración de los Ayuntamientos y reducción de sus competencias, son el escenario idóneo para la incorporación de valores sociales.

Los objetivos que entendemos se deberían buscar, en aras de una mejora e incremento de los beneficios sociales y medioambientales por parte de las entidades públicas son, sin duda, el aumento en la eficacia de la incorporación del clausulado social, así como el desarrollo de procedimientos y herramientas que puedan ser adoptados por cualquier Administración Pública, lo que permitiría reducir la carga de trabajo, especialmente en las entidades locales.

La realidad de una legislación favorable unida a un entorno socioeconómico propicio conforman un escenario adecuado para que las Administraciones Públicas hagan uso de las herramientas de las que disponen para cumplir con los objetivos sociales necesarios.

Las Administraciones disponen, por tanto, de diversas prácticas para incorporar el valor social en su contratación:

a) Incluyendo aspectos sociales en el objeto del contrato

b) Mejorando la accesibilidad de entidades socialmente responsables y/o pequeñas

c) Considerando la posibilidad de reserva

d) Eligiendo el tipo de licitación más conveniente

e) Concretando las especificaciones técnicas

f) Estableciendo los criterios de adjudicación o

g) Decidiendo condiciones de cumplimiento del contrato

En lo que se refiere a incluir aspectos sociales en el objeto del contrato, la Administración tiene la capacidad de hacerlo. Si no lo hace en esta fase, es necesario que sea muy cuidadosa al introducirlos en otros capítulos del contrato, ya que las consideraciones sociales en las especificaciones técnicas o en los criterios de adjudicación, sólo son aceptables si están vinculadas al objeto del contrato, tal y como recoge la legislación. Por otra parte, al tener que elegir siempre la oferta económica más ventajosa, podrá incluir la mejor relación calidad-precio evaluada en función de criterios que incluyan aspectos cuantitativos, medioambientales y/o sociales, es decir desde el planteamiento coste-eficacia, entendiéndolo como el cálculo del "coste del ciclo de la vida".

Cuando se habla de la mejora de la accesibilidad de entidades socialmente responsables y/o pequeñas, la Administración debe asegurarse de publicitar la licitación de forma que se garantice el conocimiento por parte 
de las entidades socialmente responsables de ese ámbito. En caso de contratos de cierto volumen, también puede contemplarse la posibilidad de fraccionamiento en lotes, para favorecer la participación de entidades de menor tamaño.

El caso de la consideración de la posibilidad de reserva es más ambiguo, ya que distintas legislaciones consideran esta facultad de diferentes maneras. Así, la Directiva 2014/24/EU reconoce la reserva a talleres protegidos, entidades con objetivos de integración sociolaboral de discapacitados y desfavorecidos y programas de empleo protegido (con más del 30\% de discapacitados o desfavorecidos). Por su parte, la Ley 30/2007 considera únicamente preferencia de adjudicación, no la reserva propiamente dicha, mientras que las legislaciones territoriales varían desde estimar la reserva (Generalitat de Catalunya) a conceder puntos extra (Navarra) o valorar más a las empresas que generan empleo estable (Comunidad de Madrid).

Sin embargo es necesario puntualizar que, a través de la Directiva sobre contratación pública 2014/24/EU se plasma la opinión de la UE de que cualquier ley nacional que prevea acceso restringido a ciertos servicios para operadores sin ánimo de lucro, supondría una restricción de los artículos 49 y 56 del Tratado de la Unión Europea sobre libertad de establecimiento y libre circulación de servicios, y debería justificarse caso por caso. Las Administraciones podrán justificar la reserva si es necesaria y proporcionada para la obtención de determinados objetivos sociales que persigue el sistema de bienestar social nacional.

La elección del tipo de licitación puede favorecer la participación de entidades de "tipo social" en la misma. Entendemos que los siguientes casos o ejemplos de contrato permitirín a la Administración la valoración óptima de aquellas empresas que impulsen valores sociales:

- Contrato menor de importe inferior a $3.050 €$ para obras y de $12.020 €$ para suministros y servicios, se adjudica sin publicidad y de forma directa, con una duración máxima de 1 año.

- Procedimiento negociado por el que se invita a no menos de tres empresas capacitadas y se negocian los términos. Por debajo de $60.101 €$ en obras y suministros y de $30.050 €$ en servicios. Se puede negociar y adjudicar el contrato sólo con empresas de inserción.

- Mercado tutelado utilizando el contrato menor y el procedimiento negociado.

- Admisión de variantes pudiendo presentarse mejoras referidas a temas sociales.

Si las entidades públicas quisieran dar peso al valor social concretando las especificaciones técnicas, éstas podrían redactarse en términos de:

- las características finales que ha de tener el producto o el servicio,

- la funcionalidad del producto o servicio,

- el proceso de producción del producto o servicio o

- la capacidad técnica del adjudicatario (habilidades, profesionalidad, experiencia, etc.). En este último caso, se pediría información sobre contratos similares realizados por el mismo para justificar la solvencia técnica, entendiendo que es mejor que el adjudicatario acredite su experiencia y/o su valor social que poner como condición que su empresa clasificada como un tipo concreto, ya que esto último puede generar mayores conflictos que la primera posibilidad propuesta.

En lo que respecta al establecimiento de los criterios de adjudicación hay que tener en cuenta las siguientes consideraciones:

1. Dar puntuación, en la valoración previa a la adjudicación, a la contratación de personas en situación desfavorecida, cuando está relacionada con el objeto del contrato y con un criterio proporcionado.

2. Entendemos que incrementar la puntuación de una empresa por ser una "empresa de inserción" es menos aconsejable, por poderse interpretar como una vulneración de los principios de igualdad y libre competencia.

3. Existe también la posibilidad de utilizar el Criterio Adicional "social". En la Directiva de Contratación Pública 2004/18 y que recoge la Ley 30/2007 de contratos del sector público, aparece esta figura, pero la administración sólo podrá utilizar este criterio de adjudicación cuando la elección haya que hacerla sobre dos ofertas idénticas en valor económico.

4. Las Administraciones podrán llevar a cabo una evaluación de los costes utilizando el concepto de coste del ciclo de vida (Concepto de la Directiva de Contratación Pública). 
5. También está en su mano incluir como criterios de adjudicación el cumplimiento de las obligaciones medioambientales, sociales o laborales establecidas en el Derecho de la Unión, Derecho nacional, convenios colectivos y otras disposiciones.

6. Y cabe la posibilidad de eliminar candidatos adjudicatarios por no cumplir normas o leyes (incluidas las de ámbito social/medioambientales).

Aquí es necesario destacar que los poderes adjudicadores podrían exigir etiquetas concretas para favorecer el inclinar la balanza del lado del beneficio social, pero han de tener en cuenta que deberán aceptar también un expediente técnico del fabricante u otro medio de prueba adecuado, siempre y cuando el operador manifieste no poder conseguirla.

Además, las posibilidades de las entidades públicas, en el caso de la decisión de condiciones de cumplimiento del contrato como herramienta para la valorización social, podrían ser: la exigencia de la contratación de un porcentaje de personas en situación de exclusión, la reserva de un determinado número de contratos, la demanda de la realización de un diseño y ejecución de un proyecto de inserción, de la contratación de un técnico de acompañamiento sociolaboral o de la emisión de informes sociolaborales.

En última instancia, interesa llevar a cabo el cálculo del "valor social de la entidad adjudicataria", que podría ser incluido entre los datos requeridos para la obtención del certificado del registro oficial de licitadores y empresas clasificadas. Con este cálculo se daría preferencia a los criterios medibles y cuantificables frente a criterios más subjetivos, de acuerdo con la nueva Directiva Europea de Contratación Pública.

\section{CONCLUSIONES}

En el presente análisis hemos puesto de manifiesto la importancia de que las Administraciones Públicas atiendan la generación de valor social en la contratación pública, y tras una revisión de la situación, principalmente en el ámbito regulatorio, planteamos algunas recomendaciones a tener en cuenta en su implementación.

En resumen, a la hora de construir propuestas para incorporar el valor social en la contratación pública, las Administraciones Públicas han de considerar los siguientes puntos de partida:

1. La posición reacia de la UE al concepto de reserva generalizada de contratos.

2. Existe la posibilidad de exigir capacidad técnica y experiencia en contratos similares.

3. Se podría homogeneizar de la contratación pública social a través de la elaboración de un Documento Europeo Único de Contratación.

4. La Legislación española reconoce la preferencia en la adjudicación del contrato, más quela reserva.

5. Se postula la no contratación de adjudicatarios que no cumplen leyes/normas (incluidas sociales/ medioambientales).

6. Existe la posibilidad de exigir "etiquetas" o información equivalente.

7. Existe un reconocimiento de la necesidad de libertad legislativa a los Estados miembros de la Unión Europea en los contratos de servicios.

8. Se puede incorporar el del concepto "coste de la vida", para cuantificar en términos monetarios los costes a lo largo del ciclo de vida de las obras, suministros y servicios.

9. Se impone dar preferencia en la valoración a los criterios medibles frente a criterios más subjetivos.

Estos ingredientes han de servir, constatada la necesidad de consecución de métodos estandarizados, utilizables por todas las Administraciones Públicas (a nivel comunitario, estatal, autonómico), para superar el actual horizonte en el que se han multiplicado las fórmulas legislativas heterogéneas respecto a la manera de incluir aspectos sociales en la contratación pública y reduciendo, de esta forma, el volumen de trabajo de las diferentes entidades, como se expuso con anterioridad.

De igual forma, con esos puntos de partida ha de buscarse el incremento de la eficacia de la contratación pública en la promoción de causas sociales para que la adjudicación de los contratos públicos recaiga sobre aquellas entidades que generen un mayor valor social.

Queda mucho camino que recorrer en la incorporación del valor social en la contratación pública, y muchos aspectos que continuar analizando sobre el tema, como seguir avanzando en el análisis comparado entre países (por ejemplo, los países nórdicos o los anglosajones) o un tema fundamental, el diseño de mecanismos de control 
que garanticen no sólo el proceso de contratación atendiendo a valor social sino la fase posterior de ejecución. La síntesis que aquí presentamos, es, pues, un primer paso, proporcionando una panorámica actual y horizontes de cambio, así como el diagnóstico y recomendaciones que extraemos, lo que creemos pueden ser de utilidad para avanzar en tan relevante camino para nuestros gobiernos y ciudadanos.

\section{BIBLIOGRAFÍA Y LEGISLACIÓN}

Alfonso, C. (2008). Plan de contratación pública verde: la compra sostenible. Ambienta: La revista del Ministerio de Medio Ambiente, (74), 55-58.

Alonso-Alegre, G. (2009). Manual de contratación pública socialmente responsable en relación con las personas con discapacidad. CERMI / FUNDACIÓN ONCE.

Araujo, A., Carter, R. Y., Callender, G., Drabkin, D., Grimm, R., Jensen, K. y Telgen, J. (2005). Challenges in Public Procurement: an international perspective. Boca Raton, FL: PrAcademics Press.

Aréjula, J. M. G. y Azurmendi, B. B. (2014). El papel de la Economía Social en la contratación pública del Gobierno vasco entre 2010 y 2012. CIRIEC-España, Revista de Economía Pública, Social y Cooperativa, (82), $207-232$.

Bernete, J. (2013). Cláusulas sociales en la contratación pública: nuevos instrumentos para el fomento del empleo a nivel local. Cuadernos de Trabajo Social, 26 (1), 85-94.

Brunete, M.T. (2010). Guía práctica - Criterios sociales y medioambientales en la contratación pública. Federación de Municipios y Provincias Castilla-La Mancha

Bovis, C. (2012). EU public procurement law. Edward Elgar Publishing.

Brammer, S. y Walker, H. (2011). Sustainable procurement in the public sector: an international comparative study. International Journal of Operations and Production Management, 31(4), 452-476.

Comisión Europea. (2010). Adquisiciones sociales. Una guía para considerar aspectos sociales en las contrataciones públicas. Luxemburgo: Dirección General de Empleo, Asuntos Sociales e Igualdad de Oportunidades.

Comunidad Autónoma de Andalucía (2003). Ley 18/2003, de 29 de diciembre, por la que se aprueban medidas fiscales y administrativas. BOJA 251, 31 de diciembre de 2003, 27502.

Comunidad Autónoma de Castilla y León (2012). Acuerdo 59/2012, de 26 de julio, de la Junta de Castilla y León, por el que se aprueban directrices vinculantes para los órganos de contratación de la Administración de la Comunidad de Castilla y León sobre incorporación de cláusulas sociales en la contratación pública. Boletín Oficial de Castilla y León, 147, 1 de agosto de 2012, 49694-49710.

Comunidad Autónoma de Cataluña (2015). Ley 3/2015, de 11 de marzo, de medidas fiscales, financieras y administrativas. BOE núm. 81, 4 de abril de 2015, 28409-28529.

Comunidad Autónoma de Extremadura (2014). Resolución de 1 de agosto de 2014, de la Consejera, por la que se dispone la publicación del Acuerdo de Consejo de Gobierno de 8 de julio de 2014, por el que se aprueban las directrices de política general dirigidas a los órganos de contratación de la Administración de la Comunidad Autónoma de Extremadura sobre contratos reservados e incorporación de cláusulas sociales en la contratación pública. DOE 150, 5 de agosto de 2014, 24372-24383.

Comunidad Autónoma del País Vasco (2008). Resolución 6/2008, de 2 de junio, del Director de la Secretaría del Gobierno y de Relaciones con el Parlamento, por la que se dispone la publicación del Acuerdo adoptado por el Consejo de Gobierno "sobre incorporación de criterios sociales, ambientales y otras políticas públicas en la contratación de la Administración de la Comunidad Autónoma y de su sector público”. BOPV 116,19 de junio de 2008, 15911-15943.

Comunidad Foral de Guipúzcoa (2013). Norma Foral 4/2013, de 17 de julio, de incorporación de cláusulas sociales en los contratos de obras del Sector Público Foral. Boletín Oficial de Guipúzcoa 139, 22 de julio de 2013, 2-16.

Comunidad Foral de Navarra (2013). Ley Foral 3/2013, de 25 de febrero, de modificación de la Ley Foral 6/2006, de 9 de junio, de Contratos Públicos. BOE 64, 15 de marzo de 2013, 20881-20916.

Comunidad Foral de Navarra (2006). Ley foral 6/2006, de 9 de junio, de contratos públicos. Boletín Oficial de Navarra, 72 de 16 de junio, 2006. BOE 64, 15 de marzo de 2013, I, 20881-20916.

Defourny, J. y Nyssens, M. (2008). Social enterprise in Europe: recent trends and developments. Social Enterprise journal, 4(3), 202-228.

Domínguez, A.P. (2013). Contratos públicos y crisis económica. Documento de Trabajo Facultad de Ciencias Sociales de Cuenca de la Universidad de Castilla-La Mancha.

Edler, J. y Georghiou, L. (2007). Public procurement and innovation - Resurrecting the demand side. Research policy, 36(7), 949-963.

Erridge, A. y Greer, J. (2002). Partnerships and public procurement: building social capital through supply relations. Public Administration, 80(3), 503-522.

Federación de Asociaciones Empresariales de Empresas de Inserción [en línea]. Clausulas sociales contratación pública y empresas de inserción. Disponible en internet: www.faedei.org. 
Generalitat de Catalunya. Departamento de Economía y Conocimiento (2012). Guía para la inclusión de cláusulas contractuales de carácter social. Versión actualizada a noviembre de 2012. Junta Consultiva de Contratación Administrativa.

Junta de Andalucía. Conserjería para la igualdad y bienestar social (2011). Dossier par a la contratación pública sostenible. Cláusulas Sociales. Fundación para la Atención e Incorporación Social.

Laville, J.-L., Lemaître, A. and Nyssens, M., 2006. Public policies and social enterprises in Europe: The challenge of institutionalization. In: Nyssens, M. ed. Social enterprise: At the crossroads of market, public policies and civil society. London: Routledge, 272-295.

McCrudden, C. (2004). Using public procurement to achieve social outcomes. In Natural Resources Forum (Vol. 28, No. 4, pp. 257-267). Blackwell Publishing Ltd.

Medina Jurado, P. (2010). Las cláusulas sociales en la contratación pública: retos y perspectivas. Cemci, 7, 2010.

Ministerio de Economía y Hacienda (2011). Real Decreto Legislativo 3/2011, de 14 de noviembre, por el que se aprueba el texto refundido de la Ley de Contratos del Sector Público. BOE276, 16 de noviembre de 2011.

Schulten, T., Alsos, K., Burgess, P. y Pedersen, K. (2012). Pay and other social clauses in European public procurement. An overview on regulation and practices with a focus on Denmark, Germany, Norway, Switzerland and the United Kingdom. European Federation on Public Service Unions [cited 13 August 2014]. Available from Internet: http://www. epsu. org/IMG/pdf/EPSU_Report_final. pdf.

Thai, K. V. (2001). Public procurement re-examined. Journal of Public Procurement, 1(1), 9-50.

Unión Europea (2004). Directiva 2004/18/CE del Parlamento Europeo y del Consejo, de 31 de marzo de 2004, sobre coordinación de los procedimientos de adjudicación de los contratos públicos de obras, de suministro y de servicios. DOUE 134, 30 de abril de 2004, 114-240.

Unión Europea (2014). Directiva 2014/24/UE del Parlamento Europeo y del Consejo, de 26 de febrero de 2014, sobre contratación pública y por la que se deroga la Directiva 2004/18/CE.DOUE94, 28 de marzo de 2014, 65-242.

Zabalegui, S. L. (2002). Manual para la aplicación de cláusulas sociales. La adjudicación de Contratos de las Administraciones Públicas como herramienta de inserción sociolaboral para personas en situación o riesgo de exclusión social. Fundación Gaztelan.

Zabalegui, S. L. (2010). Guía práctica para la incorporación de cláusulas sociales en la contratación pública. Delegación de Promoción Económica y Empleo. Área de Cultura, Juventud y Cooperación Local. Diputación de Granada. 